\title{
Downregulation of Williams syndrome transcription factor (WSTF) suppresses glioblastoma cell growth and invasion by inhibiting PI3K/AKT signal pathway
}

\author{
Liyuan Yang, Chunfu Du, Hui Chen, Zhengwen Diao \\ Department of Neurosurgery, The People's Hospital of Yaan, Sichuan, China
}

\begin{abstract}
Williams syndrome transcription factor (WSTF) participates in diverse cellular processes, including tumor cell proliferation and migration. However, the function of WSTF in glioblastoma (GBM) remains unknown. Data from the Gene Expression Profiling Interactive Analysis (GEPIA) and The Cancer Genome Atlas (TCGA) datasets showed that WSTF was upregulated in GBM tissues. Moreover, WSTF was also increased in the GBM cells. pcDNA-mediated over-expression of WSTF contributed to cell proliferation and invasion of GBM cells, while GBM cell proliferation and invasion were suppressed by shRNA-mediated silencing of WSTF. Additionally, GBM cell apoptosis was reduced by over-expression of WSTF accompanied by decrease in Bax and cleaved caspase-3, while promoted by silencing of WSTF with increase in Bax and cleaved caspase-3. Protein expression of AKT phosphorylation was enhanced by WSTF over-expression while reduced by WSTF silencing. Inhibitor of phosphatidylinositol 3 kinase attenuated WSTF over-expression-induced increase in GBM cell proliferation and invasion. In conclusion, WSTF contributed to GBM cell growth and invasion through activation of PI3K/AKT pathway.
\end{abstract}

Key words: WSTF; glioblastoma; proliferation; invasion; PI3K/AKT.

Correspondence: Liyuan Yang, Department of Neurosurgery, The People's Hospital of YAAN, No. 358, Chenghou Road, Yucheng District, Yaan, Sichuan Province, China.

Tel: +86.0835.2862065. E-mail: yangliyuan57@163.com

Contributions: LY, CD, designed the study, supervised the data collection; HC, analyzed and interpreted the data, ZD, prepared the manuscript for publication and reviewed the draft of the manuscript. All the authors have read and approved the final version of the manuscript and agreed to be accountable for all aspects of the work.

Conflict of interest: The authors declare that they have no competing interests, and all authors confirm accuracy.

Ethics approval: Not applicable.

Availability of data and materials: All data generated or analyzed during this study are included in this published article. 


\section{Introduction}

Glioblastoma (GBM) is one of the most deadliest cancers in humans. ${ }^{1}$ Proliferation and invasion are the devastating characteristics of GBM and contribute to the high mortality rate. ${ }^{1}$ A variety of clinical treatment strategies, including surgical treatment, radiation therapy combined with temozolomide chemotherapy, are currently used for the patients with GBM, and the prognosis of GBM is still very poor and the average survival time of patients is still 12-15 months. ${ }^{2}$ Novel and specific drug targets for prevention of GBM cell proliferation and invasion are urgently needed due to the limited therapeutic effects of current strategies. ${ }^{3}$

Williams syndrome transcription factor (WSTF) is encoded by bromodomain adjacent to zinc finger domain $1 \mathrm{~B}$ gene and has been identified as a hemizygous deletion gene in patients with Williams syndrome. ${ }^{4}$ WSTF, the component of WSTF-Imitation Switch (ISWI) ATP-dependent chromatin-remodeling complex, contains the $\mathrm{C}$-terminal bromine domain adjacent to plant homologous domain to participate in DNA damage through tyrosine phosphorylation of gamma-H2A.X. ${ }^{5}$ Loss of WSTF was involved in heterochromatin formation and resolution and participated in the pathogenesis of Williams-Beuren syndrome. ${ }^{6}$ WSTF was recruited to the DNA damage site induced by UV-C to specifically facilitate the transcription recovery. ${ }^{7}$ Recent studies have found that WSTF also plays a very important role in the occurrence and development of tumors. For example, estrogen receptor signaling was activated by WSTF to promote breast cancer cell growth. ${ }^{8}$ WSTF can form a complex with neuregulin-3 to activate the oncogenic pathways in colon tumor cells. ${ }^{9}$ Downregulation of gamma-H2A.X phosphorylation by mouse double minute 2 homolog-WSTF-eyes absent transcriptional coactivator and phosphatase 3 complex promoted the progression of gastric cancer. ${ }^{10}$ However, the effects of WSTF on GBM cell proliferation and invasion have not been reported until now.

In this study, the expression level of WSTF in GBM cells was firstly identified, and its effects on cell proliferation and invasion of GBM cells were then investigated. The investigation of downstream signaling pathways might provide theoretical basis for identification of WSTF as the potential therapeutic target for the prevention of GBM.

\section{Materials and Methods}

\section{Validation of WSTF}

GEPIA database (http://gepia.cancer-pku.cn/) was used to detect the expression of WSTF in TCGA RNA-seq raw data from 163 GBM tissues and 207 normal tissues.

\section{Cell culture, transfection, and treatment}

GBM cell lines (U251MG, LN-229, A172) and human microglial clone 3 cell line (HMC3) were received from ATCC (Manassas, VA, USA) and then cultured in DMEM medium with $10 \%$ fetal bovine serum (Gibco BRL, Grand Island, NY, USA) in a $37^{\circ} \mathrm{C}$ humidified chamber. pcDNA3.1-WSTF and shRNAs targeting WSTF (shWSTF-1\# or 2\#) were constructed by RiboBio (Guangzhou, China). U251MG and LN-229 cells were transfected with pcDNA vectors or shRNAs by Lipofectamine 2000 (Thermo Fisher Scientific, Waltham, MA, USA). U251MG and LN-229 under pcDNA vectors transfection were also incubated with $15 \mu \mathrm{M}$ LY294002 (Sigma-Aldrich, St. Louis, MO, USA) for $48 \mathrm{~h}$, and the cells were used for functional assays.

\section{Cell viability and proliferation}

U251MG and LN-229 cells with indicated transfection and treatment were plated in a 96-well plate for $24 \mathrm{~h}$. A total of $10 \mu \mathrm{L}$ CCK8 solution (Sigma-Aldrich) was added to each well for $2 \mathrm{~h}$ before measurement of absorbance at $450 \mathrm{~nm}$ under spectrometer (Thermo Fisher Scientific). U251MG and LN-229 cells with indicated transfection and treatment were also plated in a 6-well plate for two weeks. Formaldehyde-fixed cells were stained with $0.4 \%$ crystal violet (Sigma-Aldrich) for the measurement of colonies under a light microscope (Olympus Corp. ,Tokyo, Japan).

\section{Cell apoptosis, migration and invasion}

U251MG and LN-229 cells with indicated transfection and treatment were suspended in $100 \mu \mathrm{L}$ Annexin V-binding buffer (Thermo Fisher Scientific), and then incubated with $5 \mu \mathrm{L}$ Annexin V-FITC (Thermo Fisher Scientific). Following incubation with of $400 \mu \mathrm{L}$ Annexin V-binding buffer containing $2 \mu \mathrm{L}$ PI solution $(2$ $\mathrm{mg} / \mathrm{mL}$ ), the cell apoptosis rate was analyzed by Attune ${ }^{\mathrm{TM}}$ Flow Cytometer (Thermo Fisher Scientific). For investigation of cell migration, U251MG and LN-229 cells with indicated transfection and treatment were suspended in $200 \mu \mathrm{L}$ serum-free DMEM medium and plated in the upper chamber of well (Corning, Tewksbury, MA, USA). The lower chamber was filled with $400 \mu \mathrm{L}$ DMEM containing $10 \%$ fetal bovine serum. Twenty-four h later, the invasive cells in lower chamber were stained with $1 \%$ crystal violet and measured under the microscope (Olympus). For detection cell invasion, the well was performed with matrigel coating (BD Bioscience, San Jose, CA, USA).
A

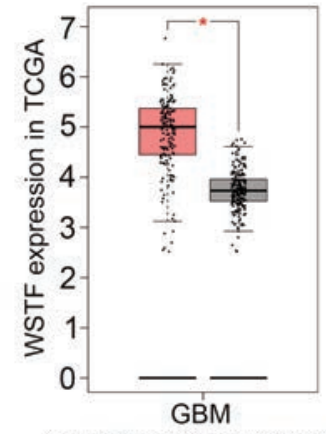

(num(T)=163; num $(N)=207$ )

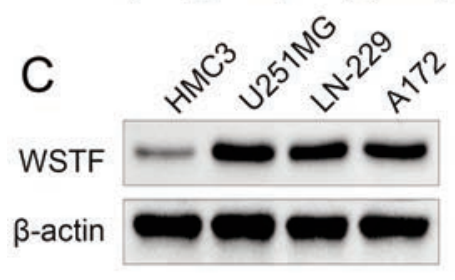

B
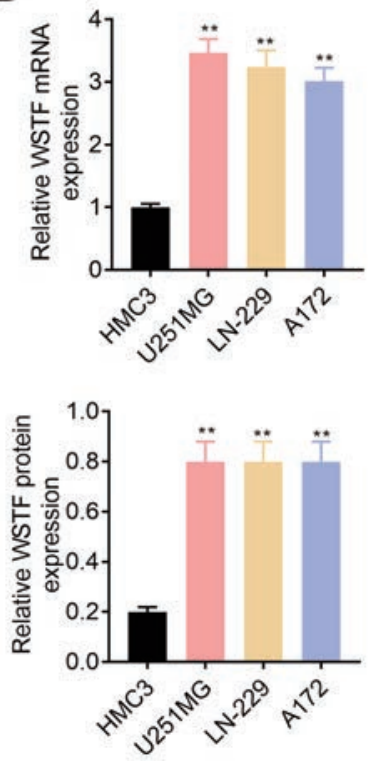

Figure 1. Upregulation of WSTF in GBM tissues and cells. A) mRNA expression of WSTF was up-regulated in GBM tissues $(n=163)$ compared to the normal tissues $(n=207)$. B) mRNA expression of WSTF was upregulation in GBM cells (U251MG, LN-229, A172) compared to human microglial clone 3 cell line (HMC3). C) Protein expression of WSTF was up-regulated in GBM cells (U251MG, LN-229, A172) compared to human microglial clone 3 cell line (HMC 3$).{ }^{*} v$ s control $\mathrm{p}<0.05$; ${ }^{* *} v s$ control $\mathbf{p}<\mathbf{0 . 0 1}$. 


\section{RT-qPCR}

RNAs were isolated from GBM cell by Trizol (Thermo Fisher Scientific), and reverse transcribed into cDNAs by MMLV reverse transcriptase (Promega, Madison, WI, USA). The mRNA expression of WSTF and GAPDH were determined via SYBR Green Master (TaKaRa, Dalian, China) with following primer sequences: GAPDH (Forward: 5'-AGGTCGGTGTGAACGGATTTG-3' and Reverse: 5'-TGTAGACCATGTAGTTGAGGTC-3') and WSTF (Forward: 5'-GGGCTCAGACACAGATGACA-3' and Reverse: 5'-TGGGGCTCAAACTTCACAAT-3').

\section{Western blot}

GBM cells were lysed in RIPA Lysis and Extraction Buffer (Thermo Fisher Scientific), and acid protein kit (Thermo Fisher Scientific) was applied to detect the protein concentrations in the cellular lysates. The lysates were separated by SDS-PAGE, electro-transferred onto PVDF membrane (Millipore, Bedford, MA, USA) and blocked in 5\% BSA. Following overnight probe with the primary antibodies: anti-WSTF (1:2000, Cell Signaling, Beverly, MA, USA), anti-Bax (1:3000, Cell Signaling), anti-cleaved caspase-3 (1:3500, Cell Signaling), anti-AKT, anti-p-AKT and anti- $\beta$ actin (1:4000, Cell Signaling), the membranes were incubated with corresponding horseradish peroxidase-labeled secondary antibody (1:5000; Cell Signaling). The immunoreactivities were detected by enhanced chemiluminescence (KeyGen, Nanjin, China).

\section{Statistical analysis}

Data with at least triple replicates were expressed as mean \pm SEM, and performed with one-way analysis of variance or
Student's $t$-test under GraphPad Prism software. The p value $<0.05$ was considered as statistically significant.

\section{Results}

\section{Upregulation of WSTF in GBM tissues and cells}

In order to evaluate WSTF expression in GBM tissues, GEPIA database (http://gepia.cancer-pku.cn/) was applied to detect TCGA RNA-seq raw data from 163 GBM tissues and 207 normal tissues. Result showed that WSTF was upregulated in GBM tissues compared with the normal tissues (Figure 1A). Both of mRNA (Figure 1B) and protein (Figure 1C) were also elevated in GBM cells (U251MG, LN-229, A172) compared with human microglial clone 3 cell line (HMC3). These results suggested that WSTF might be involved in GBM progression.

\section{WSTF contributed to GBM cell proliferation}

U251MG and LN-229 were transfected with pcDNA-WSTF or shRNAs-WSTF for gain- of loss- of functional assays. Protein expression of WSTF was enhanced in U251MG and LN-229 transfected with pcDNA-WSTF or reduced by transfection with shRNAs-WSTF (Figure 2A). pcDNA-mediated over-expression of WSTF increased cell viability of U251MG and LN-229 (Figure $2 \mathrm{~B}$ ) and promoted the cell proliferation (Figure 2C). However, the cell viability (Figure 2B) and proliferation (Figure 2C) of U251MG and LN-229 were repressed by silencing of WSTF, indicating that WSTF contributed to GBM cell proliferation.
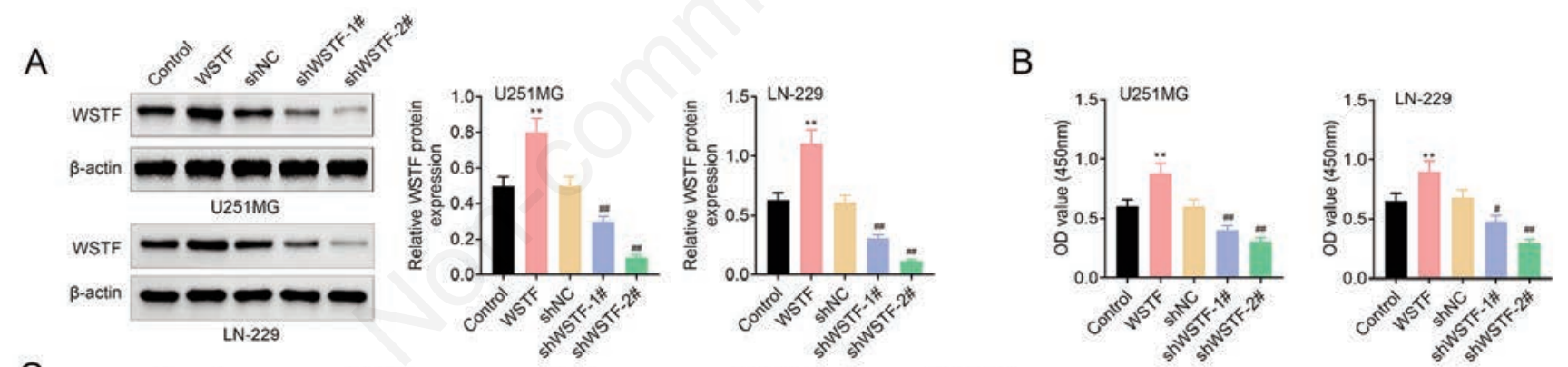

C
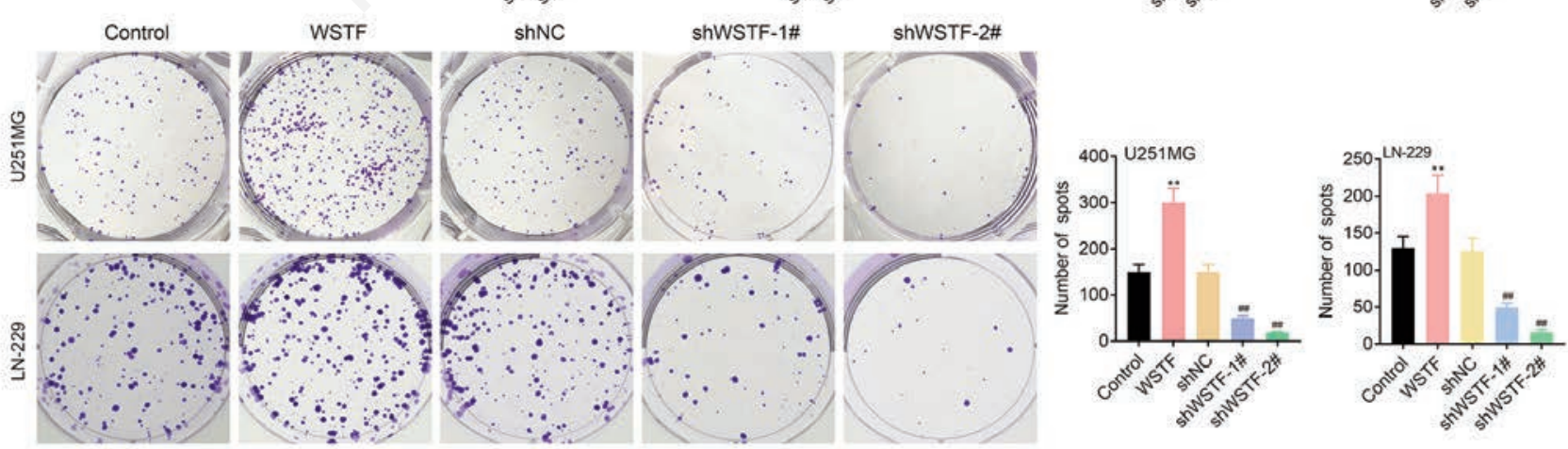

Figure 2. WSTF contributed to GBM cell proliferation. A) Protein expression of WSTF was enhanced in U251MG and LN-229 transfected with pcDNA-WSTF AND reduced by transfection with shRNAs-WSTF. B) pcDNA-mediated over-expression of WSTF increased cell viability of U251MG and LN-229, while shRNAs-mediated knockdown of WSTF suppressed the cell viability. C) pcDNA-mediated over-expression of WSTF increased cell proliferation of U251MG and LN-229, while shRNAs-mediated knockdown of WSTF suppressed the cell proliferation. ${ }^{* *} v s$ control; \#\#vs shNC $\mathrm{p}<0.01$. 


\section{Silencing of WSTF contributed to GBM cell apoptosis}

Flow cytometry analysis revealed that over-expression of WSTF suppressed cell apoptosis of U251MG and LN-229 (Figure 3A), while silencing of WSTF promoted cell apoptosis (Figure
3A). Transfection with pcDNA-WSTF decreased protein expression of Bax and cleaved caspase-3 in U251MG and LN-229 (Figure 3B), while shWSTF transfection increased the expression of Bax and cleaved caspase-3 (Figure 3B), indicating the antiapoptotic role of WSTF in GBM cell.

A

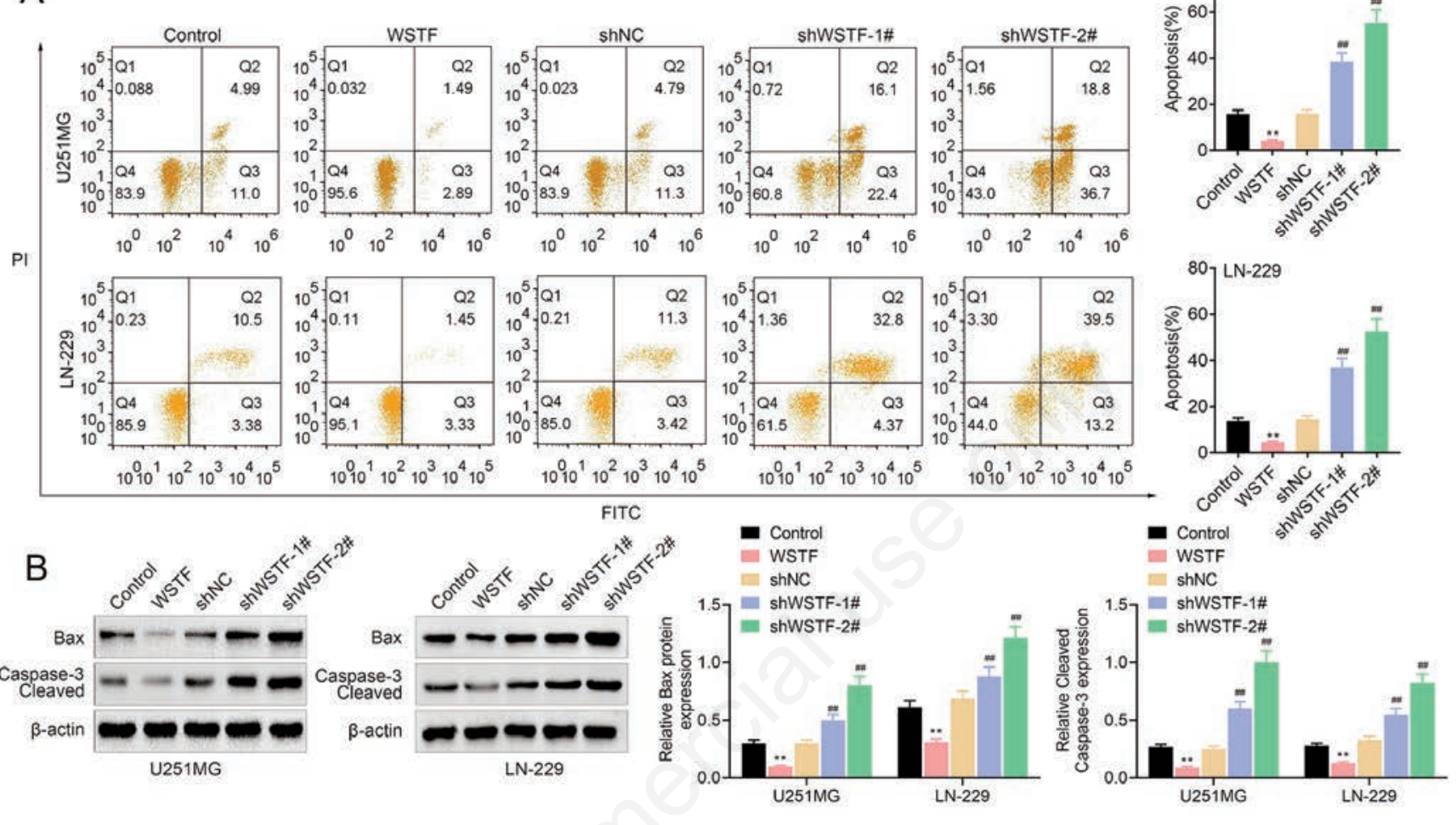

Figure 3. Silence of WSTF contributed to GBM cell apoptosis. A) pcDNA-mediated over-expression of WSTF repressed cell apoptosis of U251MG and LN-229, while shRNAs-mediated knockdown of WSTF promoted the cell apoptosis. B) pcDNA-mediated over-expression of WSTF decreased protein expression of Bax and cleaved caspase-3 in U251MG and LN-229, while shRNAs-mediated knockdown of WSTF increased the protein expression. ${ }^{* *} v s$ control; \#\#vs shNC $\mathrm{p}<0.01$.

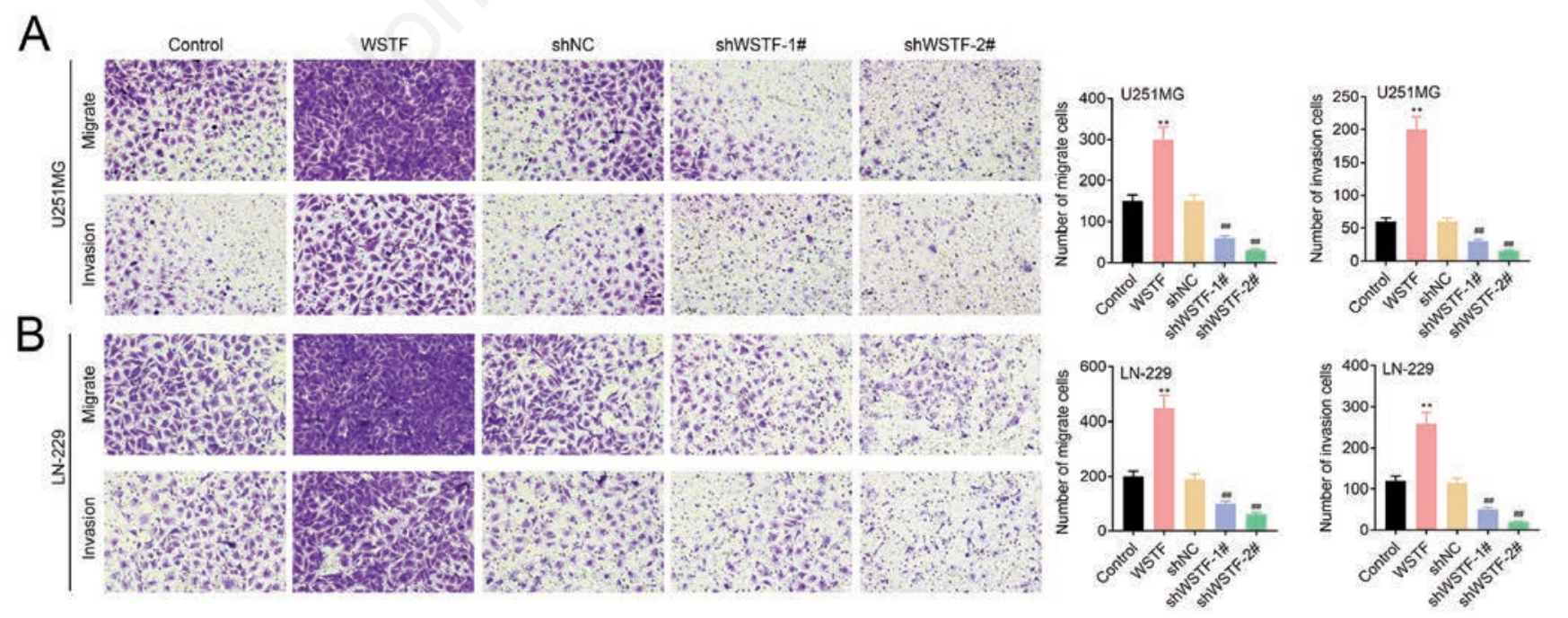

Figure 4. WSTF contributed to GBM cell invasion. pcDNA-mediated over-expression of WSTF promoted cell migration and invasion of U251MG and LN-229, while shRNAs-mediated knockdown of WSTF suppressed the cell migration and invasion. ${ }^{* *} v s$ control; \#\#vs shNC $\mathbf{p}<0.01$. 


\section{WSTF contributed to GBM cell invasion}

In addition to the proliferative and anti-apoptotic roles of WSTF in GBM cell, transwell assay was applied to assess the effect of WSTF on migration and invasion. Cell migration and invasion of U251MG and LN-229 were promoted by pcDNAmediated WSTF over-expression, whereas repressed by shRNAsmediated WSTF silencing (Figure 4), indicating the anti-invasive role of WSTF silencing in GBM cell.

\section{WSTF contributed to AKT activation in GBM cell}

Protein expression of AKT was not significantly affected by
pcDNA-mediated WSTF over-expression or shRNA-mediated WSTF silencing (Figure 5). However, AKT phosphorylation was enhanced in U251MG and LN-229 transfected with pcDNAWSTF and reduced by shWSTF transfection (Figure 5), demonstrating that WSTF contributed to AKT activation in GBM cell.

Inhibition of PI3K/AKT attenuated oncogenic role of WSTF in GBM cell

To validate whether WSTF contributed to GBM cell progression through activation of PI3K/AKT pathway, U251MG cells transfected with pcDNA-WSTF were treated with LY294002,
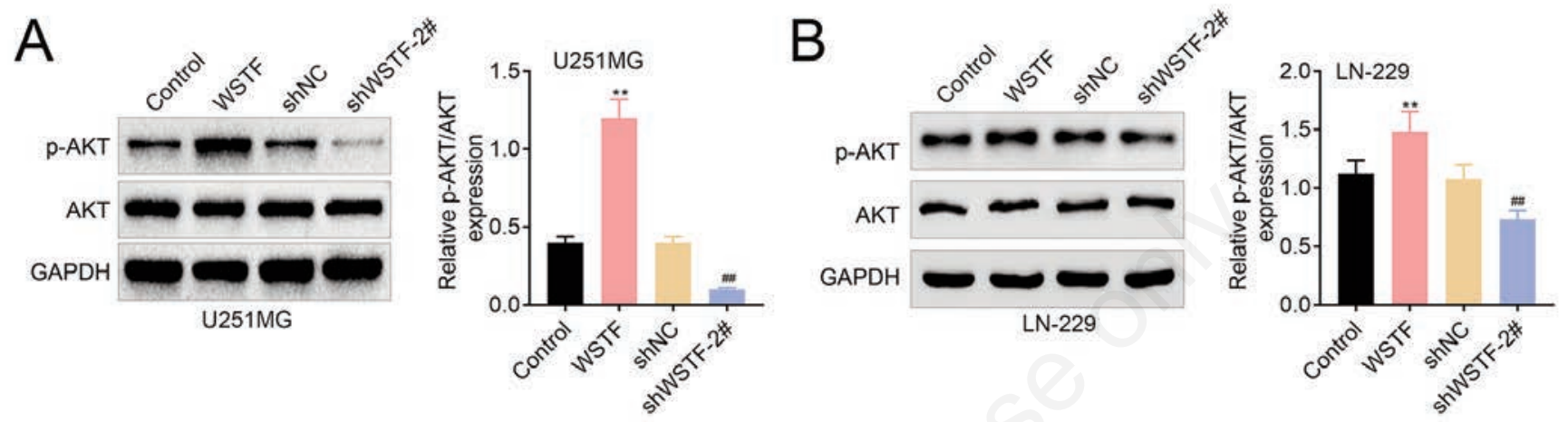

Figure 5. WSTF contributed to AKT activation in GBM cell. pcDNA-mediated over-expression of WSTF increased protein expression of p-p-AKT in U251MG (A) and LN-229 (B), while shRNAs-mediated knockdown of WSTF decreased the protein expression. **, \#\# $\mathbf{p}<\mathbf{0 . 0 1}$.

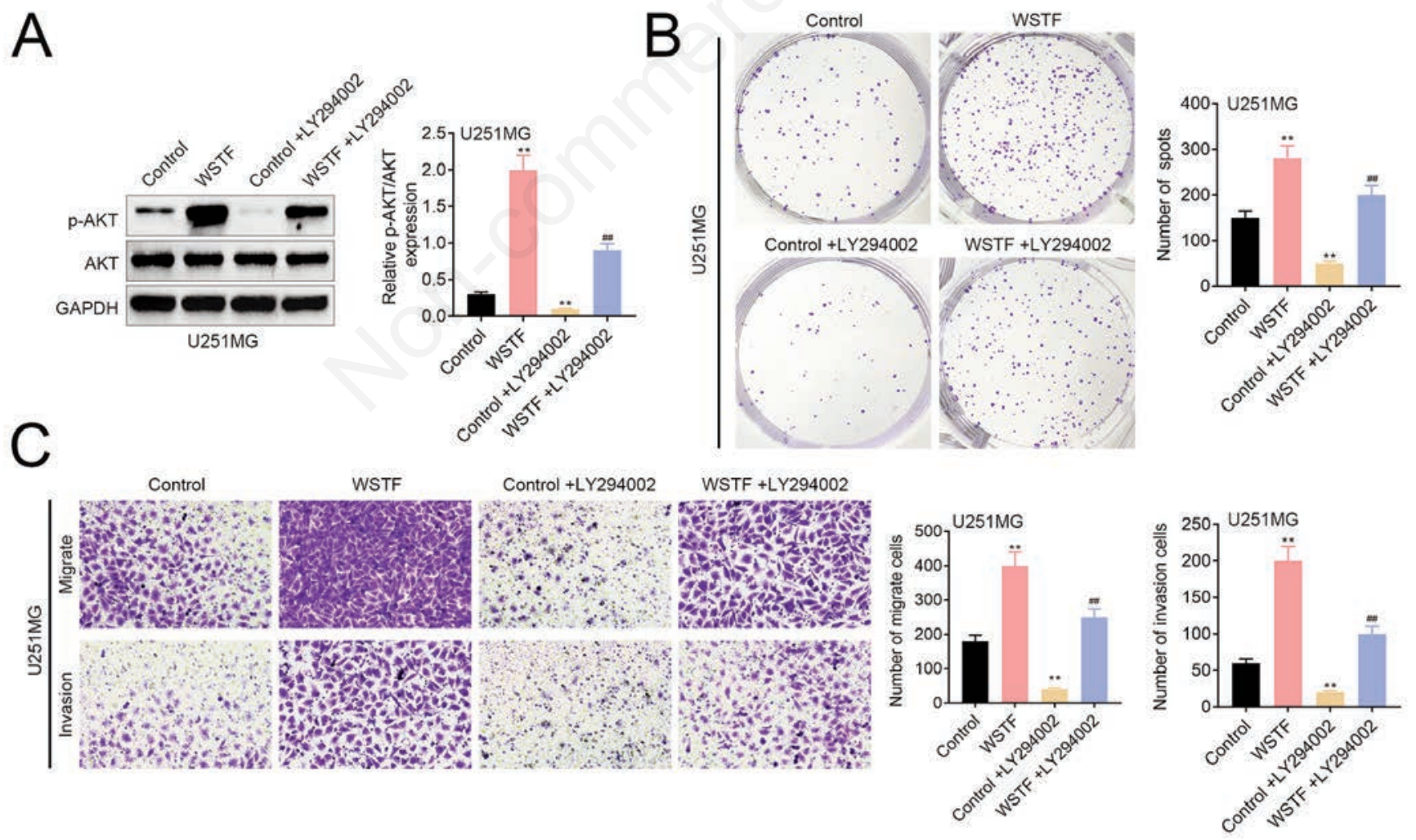

Figure 6. Inhibition of PI3K/AKT attenuated oncogenic role of WSTF in GBM cell. A) LY294002 treatment attenuated WSTF overexpression-induced increase of AKT phosphorylation in U251MG. B) LY294002 treatment attenuated WSTF over-expression-induced increase of cell proliferation in U251MG. C) LY294002 treatment attenuated WSTF over-expression-induced increase of cell migration and invasion in U251MG. ${ }^{* *} v s$ control; \#\#vs control + LY294002 p $<0.01$. 
inhibitor of PI3K. Western blot analysis showed that LY294002 treatment attenuated WSTF over-expression-induced increase in AKT phosphorylation (Figure 6A). Moreover, the cell proliferation (Figure 6B), migration and invasion (Figure 6C) induced by WSTF over-expression were also attenuated by LY294002 treatment. These results revealed that WSTF contributed to GBM cell progression through activation of PI3K/AKT pathway.

\section{Discussion}

Chemo- or radio-therapeutic agents stimulate cytotoxicity in GBM cells through DNA damage, and the DNA repair pathways could prevent the DNA damage through activation of damage sensors, such as gamma-H2A.X. ${ }^{11}$ The activation of DNA repair pathways contributes to the standard chemo- or radio-therapies. ${ }^{12}$ Therefore, checkpoint/repair molecules were involved in regulation of DNA damage repair and tumor cell apoptosis, thus participating in the pathogenesis of GBM. ${ }^{12}$ Since WSTF functions as gamma-H2A.X-binding protein to regulate DNA damage response in tumor cells, ${ }^{13}$ and WSTF activates oncogenic pathways in tumor cells, ${ }^{9}$ the regulatory role of WSTF in GBM cell growth and invasion were investigated in this study.

Bioinformatics analysis from TCGA database in this study firstly showed that mRNA expression of WSTF was enhanced in GBM tissues, and GBM cells also showed higher expression of WSTF than that of the normal control. Functional assays then indicated that over-expression of WSTF suppressed GBM cell apoptosis, and contributed to the cell proliferation, migration, and invasion. The GBM cell growth and invasion were repressed by silencing of WSTF. Considering that proliferation and invasion are the devastatingly characteristics of GBM, and suppression of GBM cell growth and invasion provided potential therapeutic targets for the treatment of GBM, ${ }^{14}$ WSTF might also be regarded as a novel therapeutic strategy for GBM. However, the in vivo suppressive role of WSTF silencing on carcinogenesis of GBM should be investigated in the further research.

A variety of signaling pathways have been reported to be implicated in GBM cell proliferation and invasion. ${ }^{15}$ $\mathrm{PI} 3 \mathrm{~K} / \mathrm{Akt} / \mathrm{mTOR}$ pathway that participates in GBM cell survival, growth, angiogenesis, invasion or drug resistance is the main therapeutic targets for drug design for GBM. ${ }^{16}$ Aberrant activation of $\mathrm{PI} 3 \mathrm{~K} / \mathrm{AKT}$ up-regulates anti-apoptotic factors and down-regulates pro-apoptotic factors to contribute to GBM cell growth. ${ }^{16}$ The inhibitors of PI3K/AKT/mTOR pathway are widely used in clinical prevention for the brain tumors, ${ }^{17}$ especially in GBM. ${ }^{18}$ Transcriptional factors, eukaryotic initiation factors, have been regarded as promising targets for glioma patients through regulation of PI3K/AKT/mTOR pathway. ${ }^{19} \mathrm{WSTF}$ promoted epithelial to mesenchymal transition of lung cancer through activation of PI3K/AKT pathway. ${ }^{20}$ Therefore, WSTF might be involved in gliomagenesis through PI3K/AKT pathway. Here, results in this study showed that protein expression of AKT phosphorylation was upregulated in GBM cells transfected with pcDNA-WSTF, while reduced by silencing of WSTF. Moreover, inhibition of PI3K attenuated WSTF over-expression-induced increase in GBM cell proliferation, migration and invasion. Therefore, WSTF might contribute to GBM cell growth and invasion through activation of PI3K/AKT pathway. Post-translational phosphorylation of WSTF at Ser158 residue regulated chromatin remodeling activity, ${ }^{21}$ and acetylation of WSTF at Lys 426 promoted the cell proliferation and invasion of tumor cells. ${ }^{22}$ The post-translational modification of WSTF involved in GBM cell progression should be investigated in the further research.

In conclusion, the present study for the first time indicated that WSTF over-expression promoted GBM cell proliferation and inva- sion through activation of PI3K/AKT pathway, and silencing of WSTF contributed to suppression of GBM progression. Therefore, WSTF might be considered as a promising therapeutic target for GBM.

\section{References}

1. Lah TT, Novak M, Breznik B. Brain malignancies: Glioblastoma and brain metastases. Semin Cancer Biol 2020;60:262-73.

2. Campos B, Olsen LR, Urup T, Poulsen HS. A comprehensive profile of recurrent glioblastoma. Oncogene 2016;35:5819-25.

3. Jiang P, Mukthavavam R, Chao Y, Bharati IS, Fogal V, Pastorino S, et al. Novel anti-glioblastoma agents and therapeutic combinations identified from a collection of FDA approved drugs. J Transl Med 2014;12:13.

4. Cho KS, Elizondo LI, Boerkoel CF. Advances in chromatin remodeling and human disease. Curr Opin Genet Dev 2004;14:308-15.

5. Xiao A, Li H, Shechter D, Ahn SH, Fabrizio LA, ErdjumentBromage $\mathrm{H}$, et al. WSTF regulates the H2A.X DNA damage response via a novel tyrosine kinase activity. Nature 2009;457:57-62.

6. Culver-Cochran AE, Chadwick BP. Loss of WSTF results in spontaneous fluctuations of heterochromatin formation and resolution, combined with substantial changes to gene expression. BMC Genomics 2013;14:740.

7. Aydin ÖZ, Marteijn JA, Ribeiro-Silva C, Rodríguez López A, Wijgers N, Smeenk G, et al. Human ISWI complexes are targeted by SMARCA5 ATPase and SLIDE domains to help resolve lesion-stalled transcription. Nucleic Acids Res 2014;42:8473-85.

8. Lundqvist J, Kirkegaard T, Laenkholm A-V, Duun-Henriksen AK, Bak M, Feldman D, et al. Williams syndrome transcription factor (WSTF) acts as an activator of estrogen receptor signaling in breast cancer cells and the effect can be abrogated by $1 \alpha, 25$-dihydroxyvitamin D3. J Steroid Biochem Mol Biol 2018;177:171-8.

9. Liu Y, Wang S-Q, Long Y-H, Chen S, Li Y-F, Zhang J-H. KRASG12 mutant induces the release of the WSTF/NRG3 complex, and contributes to an oncogenic paracrine signaling pathway. Oncotarget 2016;7:53153-64.

10. Dong C, Sun J, Ma S, Zhang G. K-ras-ERK1/2 down-regulates H2A.X(Y142ph) through WSTF to promote the progress of gastric cancer. BMC Cancer 2019;19:530.

11. Alifieris C, Trafalis DT. Glioblastoma multiforme: Pathogenesis and treatment. Pharmacol Ther 2015;152:63-82.

12. Annovazzi L, Caldera V, Mellai M, Riganti C, Battaglia L, Chirio D, et al. The DNA damage/repair cascade in glioblastoma cell lines after chemotherapeutic agent treatment. Int $\mathrm{J}$ Oncol 2015;46:2299-308.

13. Yang S, Quaresma AJC, Nickerson JA, Green KM, Shaffer SA, Imbalzano AN, et al. Subnuclear domain proteins in cancer cells support the functions of RUNX2 in the DNA damage response. J Cell Sci 2015;128:728-40.

14. Chen W-L, Turlova E, Sun CLF, Kim J-S, Huang S, Zhong X, et al. Xyloketal B suppresses glioblastoma cell proliferation and migration in vitro through inhibiting TRPM7-regulated PI3K/Akt and MEK/ERK signaling pathways. Mar Drugs 2015;13:2505-25.

15. Balça-Silva J, Matias D, Carmo Ad, Sarmento-Ribeiro AB, Lopes MC, Moura-Neto V. Cellular and molecular mechanisms of glioblastoma malignancy: Implications in resistance and therapeutic strategies. Semin Cancer Biol. 2019;58:130-41.

16. Shahcheraghi S, Tchokonte-Nana V, Lotfi M, Lotfi M, 
Ghorbani A, Sadeghnia H. Wnt/beta-catenin and PI3K/Akt/mTOR Signaling Pathways in Glioblastoma: Two Main Targets for Drug Design: A Review. Curr Pharm Design. 2020;26.

17. Crespo Pomar S, Arcaro A. The role of the PI3K/AKT/mTOR pathway in brain tumor metastasis. J Cancer Metastasis Treat 2016;2:80-9.

18. Westhoff M-A, Karpel-Massler G, Brühl O, Enzenmüller S, La Ferla-Brühl K, Siegelin MD, et al. A critical evaluation of PI3K inhibition in glioblastoma and neuroblastoma therapy. Mol Cell Ther 2014;2:32.

19. Krassnig S, Wohlrab C, Golob-Schwarzl N, Raicht A, Haybaeck J. A profound basic characterization of eIFs in gliomas: Identifying eIF3I and $4 \mathrm{H}$ as Potential novel target candidates in glioma therapy. Cancers 2021;13:1482.

20. Meng J, Zhang X-T, Liu X-L, Fan L, Li C, Sun Y, et al. WSTF promotes proliferation and invasion of lung cancer cells by inducing EMT via PI3K/Akt and IL-6/STAT3 signaling pathways. Cell Signal 2016;28:1673-82.

21. Oya H, Yokoyama A, Yamaoka I, Fujiki R, Yonezawa M, Youn $\mathrm{M}-\mathrm{Y}$, et al. Phosphorylation of Williams syndrome transcription factor by MAPK induces a switching between two distinct chromatin remodeling complexes. J Biol Chem 2009;284: 32472-82.

22. Liu Y, Zhang Y-Y, Wang S-Q, Li M, Long Y-H, Li Y-F, et al. WSTF acetylation by MOF promotes WSTF activities and oncogenic functions. Oncogene 2020;39:5056-67.

Received for publication: 25 March 2021. Accepted for publication: 23 October 2021.

This work is licensed under a Creative Commons Attribution-NonCommercial 4.0 International License (CC BY-NC 4.0).

(C) Copyright: the Author(s), 2021

Licensee PAGEPress, Italy

European Journal of Histochemistry 2021; 65:3255

doi:10.4081/ejh.2021.3255 